\title{
The Characterization of the Variational Minimizers for Spatial Restricted $N+1$-Body Problems
}

\author{
Fengying Li, Shiqing Zhang, and Xiaoxiao Zhao \\ Yangtze Center of Mathematics and College of Mathematics, Sichuan University, Chengdu 610064, China \\ Correspondence should be addressed to Fengying Li; lify0308@163.com
}

Received 8 February 2013; Accepted 27 April 2013

Academic Editor: Maoan Han

Copyright (C) 2013 Fengying Li et al. This is an open access article distributed under the Creative Commons Attribution License, which permits unrestricted use, distribution, and reproduction in any medium, provided the original work is properly cited.

\begin{abstract}
We use Jacobi's necessary condition for the variational minimizer to study the periodic solution for spatial restricted $N+1$-body problems with a zero mass on the vertical axis of the plane for $N$ equal masses. We prove that the minimizer of the Lagrangian action on the anti-T/ 2 or odd symmetric loop space must be a nonconstant periodic solution for any $2 \leq N \leq 472$; hence the zero mass must oscillate, so that it cannot be always in the same plane with the other bodies. This result contradicts with our intuition that the small mass should always be at the origin.
\end{abstract}

\section{Introduction and Main Result}

The Newtonian n-body problem [1] is a classical problem. Spatial restricted 3-body model was studied by Sitnikov [2]. Mathlouthi [3] et al. studied the periodic solutions for the spatial circular restricted 3-body problems by mini-max variational methods.

In this paper, we study spatial circular restricted $N+1$ body problems with a zero mass on the vertical axis of the plane for $N$ equal masses. Suppose point masses $m_{1}=\cdots=$ $m_{N}=1$ move on a circular orbit around the center of masses. The motion for the zero mass is governed by the gravitational forces of $m_{1}, \ldots, m_{N}$. Let $\rho_{j}=e^{\sqrt{-1}(2 \pi j / N)}$ and

$$
\begin{aligned}
q_{1}(t) & =r e^{\sqrt{-1} \pi t} \rho_{1}, \ldots, q_{j}(t) \\
& =\rho_{j} q_{1}(t), \ldots, q_{N}(t)=r e^{\sqrt{-1} \pi t}
\end{aligned}
$$

satisfy the Newtonian equations:

$$
m_{i} \ddot{q}_{i}=\frac{\partial U}{\partial q_{i}}, \quad i=1, \ldots, N,
$$

where

$$
U=\sum_{1 \leq i<j \leq N} \frac{m_{i} m_{j}}{\left|q_{i}-q_{j}\right|} .
$$

The orbit $q(t)=(0,0, z(t)) \in R^{3}$ for zero mass satisfies the following equation:

$$
\ddot{q}=\sum_{i=1}^{N} \frac{m_{i}\left(q_{i}-q\right)}{\left|q_{i}-q\right|^{3}} .
$$

Obviously, $\widetilde{q}(t)=(0,0,0)$ satisfies $(4)$; it seems that $\widetilde{q}(t) \equiv$ 0 is a variational minimizer, but we will prove it is not this is the goal of this paper.

Define

$$
f(q)=\int_{0}^{1}\left[\frac{1}{2}|\dot{q}|^{2}+\sum_{i=1}^{N} \frac{1}{\left|q-q_{i}\right|}\right] d t, \quad q \in \Lambda_{i},
$$

then

$$
f(q)=\int_{0}^{1}\left[\frac{1}{2}\left|z^{\prime}\right|^{2}+\frac{N}{\sqrt{r^{2}+z^{2}}}\right] d t \triangleq f(z), \quad q \in \Lambda_{i},
$$


where

$$
\begin{gathered}
\Lambda_{1}=\left\{\begin{array}{c}
\left.q(t)=(0,0, z(t)) \mid z(t) \in W^{1,2}\left(\frac{R}{Z}, R\right)\right\}, \\
q(-t)=-q(t)
\end{array}\right\} \\
\Lambda_{2}=\left\{\begin{array}{c}
q(t)=(0,0, z(t)) \mid z(t) \in W^{1,2}\left(\frac{R}{Z}, R\right) \\
z\left(t+\frac{1}{2}\right)=-z(t)
\end{array}\right\}, \\
W^{1,2}\left(\frac{R}{Z}, R\right)=\left\{\begin{array}{c}
x(t) \mid \begin{array}{c}
x(t), \dot{x}(t) \in L^{2}((0,1), R) \\
x(t+1)=x(t)
\end{array}
\end{array}\right\} .
\end{gathered}
$$

Notice that the symmetry in $\Lambda_{2}$ is related to the Italian symmetry [4].

In this paper, our main result is the following.

Theorem 1. The minimizer of $f(q)$ on the closure $\bar{\Lambda}_{i}$ of $\Lambda_{i}(i=$ $1,2)$ is a nonconstant periodic solution for $2 \leq N \leq 472$; hence the zero mass must oscillate, so that it can not be always in the same plane with the other bodies.

\section{Proof of Theorem 1}

We define the inner product and equivalent norm of $W^{1,2}(R / Z, R)$ :

$$
\begin{gathered}
\langle u, v\rangle=\int_{0}^{1}\left(u v+u^{\prime} \cdot v^{\prime}\right) d t \\
\|u\|=\left[\int_{0}^{1}|u|^{2} d t\right]^{1 / 2}+\left[\int_{0}^{1}\left|u^{\prime}\right|^{2} d t\right]^{1 / 2},
\end{gathered}
$$

which is equivalent to

$$
\left[\int_{0}^{1}\left|u^{\prime}\right|^{2} d t\right]^{1 / 2}+|u(0)| .
$$

Lemma 2 (Palais' Symmetry Principle [5]). By Palais' Symmetry Principle, we know that the critical point of $f(q)$ in $\bar{\Lambda}_{i}$ is a noncollission periodic solution of Newtonian equation (4).

Let $\sigma$ be an orthogonal representation of a finite or compact group $G$ in the real Hilbert space $H$ such that for all $\sigma \in G, f(\sigma$. $x)=f(x)$, where $f: H \rightarrow R$.

Let $S=\{x \in H \mid \sigma \cdot x=x$, for all $\sigma \in G\}$. Then the critical point of $f$ in $S$ is also a critical point of $f$ in $H$. lemmas:

In order to prove Theorem 1, we need the following

Lemma 3 (see [6]). Let $X$ be a reflexive Banach space, $S$ be a weakly closed subset of $X, f: S \rightarrow R \cup\{+\infty\}$, and $f \neq \equiv+\infty$ is weakly lower semicontinuous and coercive $(f(x) \rightarrow+\infty$ as $\|x\| \rightarrow+\infty)$; then $f$ attains its infimum on $S$.

Lemma 4 (Poincare-Wirtinger Inequality). Let $q \in W^{1,2}$ $\times\left(R / Z, R^{N}\right)$ and $\int_{0}^{T} q(t) d t=0$; then

$$
\int_{0}^{T}|\dot{q}(t)|^{2} d t \geq\left(\frac{2 \pi}{T}\right)^{2} \int_{0}^{T}|q(t)|^{2} d t
$$

Lemma 5. $f(q)$ in (6) attains its infimum on $\bar{\Lambda}_{1}=\Lambda_{1}$ or $\bar{\Lambda}_{2}=$ $\Lambda_{2}$.

Proof. By Lemmas 3 and 4, it is easy to prove Lemma 5.

Lemma 6 (Jacobi's Necessary Condition [7]). If the critical point $u=\widetilde{u}(t)$ corresponds to a minimum of the functional $\int_{a}^{b} F\left(t, u(t), u^{\prime}(t)\right) d t$ and if $F_{u^{\prime} u^{\prime}}>0$ along this critical point, then the open interval $(a, b)$ contains no points conjugate to $a$; that is, for all $c \in(a, b)$, the following boundary value problem

$$
\begin{gathered}
-\frac{d}{d t}\left(P h^{\prime}\right)+Q h=0, \\
h(a)=0, \quad h(c)=0,
\end{gathered}
$$

has only the trivial solution $h(t) \equiv 0$, for all $t \in(a, c)$, where

$$
\begin{gathered}
P=\left.\frac{1}{2} F_{u^{\prime} u^{\prime}}\right|_{u=\widetilde{u}} \\
Q=\left.\frac{1}{2}\left(F_{u u}-\frac{d}{d t} F_{u u^{\prime}}\right)\right|_{u=\tilde{u}} .
\end{gathered}
$$

Remark 7. It is easy to see that Lemma 6 is suitable for the fixed end problem. In this paper, we consider the periodic solutions of (2) on $\bar{\Lambda}_{i}=\Lambda_{i}(i=1,2)$; hence we need to establish a similar conclusion as Lemma 6 for the periodic boundary problem.

Lemma 8. Let $F \in C^{3}(R \times R \times R, R)$. Assume that $u=\widetilde{u}(t)$ is a critical point of the functional $\int_{0}^{T} F\left(t, u(t), u^{\prime}(t)\right) d t$ on $\left\{u \in W^{1,2}(R / T Z, R), u^{\prime}(0)=0\right\}$ and $\left.F_{u^{\prime} u^{\prime}}\right|_{u=\widetilde{u}}>0$. If the open interval $(0, T)$ contains a point $c$ conjugate to 0 , then $u=\widetilde{u}(t)$ is not a minimum of the functional $\int_{0}^{T} F\left(t, u(t), u^{\prime}(t)\right) d t$.

Proof. Suppose $u=\widetilde{u}(t)$ is a minimum of the functional $\int_{0}^{T} F\left(t, u(t), u^{\prime}(t)\right) d t$. The second variation of $\int_{0}^{T} F\left(t, u(t), u^{\prime}(t)\right) d t$ is

$$
\int_{0}^{T}\left(P h^{\prime 2}+Q h^{2}\right) d t
$$

where

$$
\begin{gathered}
P=\left.\frac{1}{2} F_{u^{\prime} u^{\prime}}\right|_{u=\tilde{u}} \\
Q=\left.\frac{1}{2}\left(F_{u u}-\frac{d}{d t} F_{u u^{\prime}}\right)\right|_{u=\tilde{u}} .
\end{gathered}
$$

Set

$$
Q_{\tilde{u}}(h)=\int_{0}^{T}\left(P h^{\prime 2}+Q h^{2}\right) d t .
$$

For all $h \in\left\{u \in W^{1,2}(R / T Z, R), u^{\prime}(0)=0\right\}$, it is easy to see that $Q_{\tilde{u}}(h) \geq 0$. Then by $Q_{\tilde{u}}(\theta)=0, \theta$ is a minimum of $Q_{\tilde{u}}(h)$. The Euler-Lagrange equation which is called the Jacobi equation of (15) is

$$
-\frac{d}{d t}\left(P h^{\prime}\right)+Q h=0 .
$$


Since the interval $(0, T)$ contains a point $c$ conjugate to 0 , there exists a nonzero Jacobi field $h_{0} \in C^{2}([0, T], R)$ satisfying

$$
\begin{gathered}
-\frac{d}{d t}\left(P h_{0}^{\prime}\right)+Q h_{0}=0, \\
h_{0}(0)=0, \quad h_{0}(c)=0, \quad h_{0}^{\prime}(0)=0 .
\end{gathered}
$$

Letting

$$
\widehat{h}(t)= \begin{cases}h_{0}(t) & t \in[0, c], \\ 0 & t \in(c, T]\end{cases}
$$

we have $\widehat{h} \in C^{2}([0, T] \backslash\{c\}, R), \widehat{h}(0)=\widehat{h}(c)=\widehat{h}(T)=0$ and

$$
\begin{aligned}
Q_{\widetilde{u}}(\widehat{h}) & =\int_{0}^{T}\left(P \widehat{h}^{\prime 2}+Q \widehat{h}^{2}\right) d t \\
& =\int_{0}^{c}\left(P h_{0}^{\prime 2}+Q h_{0}^{2}\right) d t=0 .
\end{aligned}
$$

Notice that we can extend $\widehat{h}$ periodically when we take $T$ as the period, so $\widehat{h} \in W_{0}^{1,2}(R / T Z, R)$. For all $h \in C_{0}^{1}([0, T], R)$, it is easy to check that $Q_{\tilde{u}}(h) \geq 0$. Then by (19), one has $\widehat{h} \in$ $C^{2}([0, T] \backslash\{c\}, R) \cap W_{0}^{1,2}(R / T Z, R)$ is a minimum of $Q_{\tilde{u}}(h)$. Hence we get

$$
-\frac{d}{d t}\left(P \widehat{h}^{\prime}\right)+Q \widehat{h}=0
$$

Combining with $\widehat{h}(0)=\widehat{h}(c)=0$ and $\widehat{h}^{\prime}(0)=0$, by the uniqueness of initial value problems for secondorder differential equation, we have $\widehat{h}(t) \equiv 0$ on $[0, c]$, which contradicts the definition of $\widehat{h}$. Therefore, Lemma 8 holds.

Lemma 9. The radius $r$ for the moving orbit of $N$ equal masses is

$$
r=\left(\frac{1}{4 \pi}\right)^{2 / 3}\left[\sum_{1 \leq j \leq N-1} \csc \left(\frac{\pi}{N} j\right)\right]^{1 / 3} .
$$

Proof. By (1)-(3), we have

$$
\ddot{q}_{N}=\sum_{j \neq N} \frac{q_{j}-q_{N}}{\left|q_{j}-q_{N}\right|^{3}} .
$$

Substituting (1) into (22), we have

$$
\begin{gathered}
-4 \pi^{2}=\sum_{j \neq N} \frac{\rho_{j}-\rho_{N}}{r^{3}\left|\rho_{j}-\rho_{N}\right|^{3}}, \\
4 \pi^{2} r^{3}=\sum_{j \neq N} \frac{1-\rho_{j}}{\left|1-\rho_{j}\right|^{3}}=\frac{1}{4} \sum_{1 \leq j \leq N-1} \csc \left(\frac{\pi}{N} j\right) .
\end{gathered}
$$

Then

$$
r^{3}=\frac{1}{16 \pi^{2}} \sum_{1 \leq j \leq N-1} \csc \left(\frac{\pi}{N} j\right)
$$

Therefore

$$
r=\left(\frac{1}{4 \pi}\right)^{2 / 3}\left[\sum_{1 \leq j \leq N-1} \csc \left(\frac{\pi}{N} j\right)\right]^{1 / 3} .
$$

Proof of Theorem 1. Clearly, $q(t)=(0,0,0)$ is a critical point of $f(q)$ on $\bar{\Lambda}_{i}=\Lambda_{i}(i=1,2)$. For the functional (6), let

$$
F\left(z, z^{\prime}\right)=\frac{1}{2}\left|z^{\prime}\right|^{2}+\frac{N}{\sqrt{r^{2}+z^{2}}} .
$$

Then the second variation of (6) in the neighborhood of $z=0$ is given by

$$
\int_{0}^{1}\left(P h^{\prime 2}+Q h^{2}\right) d t
$$

where

$$
\begin{gathered}
P=\left.\frac{1}{2} F_{z^{\prime} z^{\prime}}\right|_{z=0}=\frac{1}{2}, \\
Q=\left.\frac{1}{2}\left(F_{z z}-\frac{d}{d t} F_{z z^{\prime}}\right)\right|_{z=0}=-\frac{N}{2 r^{3}} .
\end{gathered}
$$

The Euler equation of (27) is called the Jacobi equation of the original functional (6), which is

$$
-\frac{d}{d t}\left(P h^{\prime}\right)+Q h=0,
$$

that is,

$$
h^{\prime \prime}+\frac{N}{r^{3}} h=0 .
$$

Next, we study the solution of (30) with initial values $h(0)=$ $0, h^{\prime}(0)=1$. It is easy to get

$$
h(t)=\sqrt{\frac{r^{3}}{N}} \cdot \sin \sqrt{\frac{N}{r^{3}}} t,
$$

which is not identically zero on $[0,1]$, but we will prove $h(c)=$ 0 for some $c \in(0,1)$.

Suppose there exists $c \in(0,1)$ such that $h(c)=0$.

Hence, for some $k \in Z^{+}$

$$
\sqrt{\frac{N}{r^{3}}} c=k \pi
$$

We have

$$
\begin{aligned}
c & =\left[\frac{\pi^{2} k^{2} r^{3}}{N}\right]^{1 / 2} \\
& =\left[\frac{k^{2}\left(\sum_{j=1}^{N-1} \csc ((\pi / N) j)\right)}{16 N}\right]^{1 / 2}
\end{aligned}
$$


Case 1 (Minimizing $f(q)$ on $\bar{\Lambda}_{1}=\Lambda_{1}$ ). Letting $0<c<1 / 2$,

$$
\bar{h}(t)= \begin{cases}h(t) & t \in[0, c], \\ 0 & t \in(c, 1-c], \\ -h(1-t) & t \in(1-c, 1] .\end{cases}
$$

It is easy to check that $\bar{h}(t) \in C^{2}([0,1] \backslash\{c, 1-c\}, R) \cap$ $W^{1,2}(R, R), \bar{h}(-t)=-\bar{h}(t), \bar{h}(0)=h(0)=0, \bar{h}(c)=h(c)=0$, and $\bar{h}$ is a nonzero solution of (30).

If we take $k=1$,

$$
c=\left[\frac{\sum_{j=1}^{N-1} \csc ((\pi / N) j)}{16 N}\right]^{1 / 2}<\frac{1}{2} .
$$

It is equivalent to

$$
\sum_{j=1}^{N-1} \csc \left(\frac{\pi}{N} j\right)<4 N
$$

Let

$$
f(x)=\sum_{j \neq x} \csc \left(\frac{\pi}{x} j\right)
$$

It is not hard to check that $f(x)$ is nonmonotone. But for $2 \leq$ $N \leq 472$, (36) holds by writing program to calculate it.

Therefore, for $2 \leq N \leq 472$, we have $c \in(0,1)$ such that

$$
\sin \sqrt{\frac{N}{r^{3}}} c=\sin \pi=0 .
$$

Notice that we can extend $\bar{h}$ periodically when we take 1 as the period, so $\bar{h} \in \Lambda_{1}$. Then by Lemma $8, q(t)=(0,0,0)$ is not a local minimum for $f(q)$ on $\Lambda_{1}$. Hence the minimizers of $f(q)$ on $\Lambda_{1}$ are not always at the center of masses; they must oscillate periodically on the vertical axis; that is, the minimizers are not always coplanar with the other bodies; therefore, we get the nonplanar periodic solutions.

Case 2 (Minimizing $f(q)$ on $\bar{\Lambda}_{2}=\Lambda_{2}$ ). Let

$$
\widetilde{h}(t)= \begin{cases}h(t) & t \in[0, c], \\ 0 & t \in\left(c, \frac{1}{2}\right], \\ -h\left(t-\frac{1}{2}\right) & t \in\left(\frac{1}{2}, \frac{1}{2}+c\right], \\ 0 & t \in\left(\frac{1}{2}+c, 1\right] .\end{cases}
$$

It is easy to check that $\widetilde{h}(t) \in C^{2}([0,1] \backslash\{c, 1 / 2,(1 / 2)+c\}, R) \cap$ $W^{1,2}(R, R), \widetilde{h}(t+(1 / 2))=-\widetilde{h}(t), \widetilde{h}(0)=h(0)=0, \widetilde{h}(c)=$ $h(c)=0$, and $\widetilde{h}$ is a nonzero solution of (30).

We hope $c \in(0,1 / 2)$; that is,

$$
c=\left[\frac{\sum_{j=1}^{N-1} \csc ((\pi / N) j)}{16 N}\right]^{1 / 2}<\frac{1}{2} .
$$

It implies that

$$
\sum_{j=1}^{N-1} \csc \left(\frac{\pi}{N} j\right)<4 N
$$

Calculated by program, for $2 \leq N \leq N_{0}=472$, we have $c \in(0,1 / 2)$ such that $h(c)=0$.

Notice that we can extend $\widetilde{h}$ periodically when we take 1 as the period, so $\widetilde{h} \in \Lambda_{2}$. Then by Lemma $8, q(t)=(0,0,0)$ is not a local minimum for $f(q)$ on $\Lambda_{2}$. Hence the minimizers of $f(q)$ on $\Lambda_{2}$ are not always at the center of masses; they must oscillate periodically on the vertical axis; that is, the minimizers are not always coplanar with the other bodies; therefore, we get the nonplanar periodic solutions.

We can use another argument to get much larger $N_{0}$. We construct a test function $z(t)$ such that $f(z)<f(0)=N / r$ for $N \leq N_{0}$, where $N_{0}$ is a very large number. Let

$$
z(t)= \begin{cases}t & t \in\left[0, \frac{1}{4}\right], \\ \frac{1}{4} & t \in\left[\frac{1}{4}, \frac{1}{2}\right],\end{cases}
$$

and we extend $z(t)$ by $z(t+(1 / 2))=-z(t)$. We have

$$
\begin{aligned}
f(z) & =\frac{1}{4}+2 \int_{0}^{1 / 2} \frac{N}{\sqrt{r^{2}+z^{2}}} d t \\
& =\frac{1}{4}+2 \int_{0}^{1 / 4} \frac{N}{\sqrt{r^{2}+t^{2}}} d t+2 \int_{1 / 4}^{1 / 2} \frac{N}{\sqrt{r^{2}+(1 / 16)}} d t \\
& =\frac{1}{4}+2 N \ln \frac{1+\sqrt{1+16 r^{2}}}{4 r}+\frac{2 N}{\sqrt{1+16 r^{2}}} .
\end{aligned}
$$

Writing program to calculate, we find an $N_{0}=9 * 10^{10}$ such that $f(z)<f(0)$.

Hence $q(t)=(0,0,0)$ is not a local minimum for $f(q)$ on $\bar{\Lambda}_{i}=\Lambda_{i}(i=1,2)$. So the minimizers of $f(q)$ on $\Lambda_{i}$ are not always at the center of masses; they must oscillate periodically on the vertical axis; that is, the minimizers are not always coplanar with the other bodies; hence, we get the nonplanar periodic solutions.

\section{Acknowledgments}

The authors would like to thank the referee for his/her many valuable comments and suggestions. This paper is Supported by the national Natural Science Foundation of China (11071175) and the Ph.D Programs Foundation of Ministry of Education of China.

\section{References}

[1] A. Wintner, The Analytical Foundations of Celestial Mechanics, vol. 5 of Princeton Mathematical Series, Princeton University Press, Princeton, NJ, USA, 1941.

[2] K. Sitnikov, "Existence of oscillating motions for the three-body problem," Doklady Akademii Nauk USSR, vol. 133, pp. 303-306, 1960. 
[3] S. Mathlouthi, "Periodic orbits of the restricted three-body problem," Transactions of the American Mathematical Society, vol. 350, no. 6, pp. 2265-2276, 1998.

[4] U. Bessi and V. Coti Zelati, "Symmetries and noncollision closed orbits for planar N-body-type problems," Nonlinear Analysis. Theory, Methods \& Applications, vol. 16, no. 6, pp. 587-598, 1991.

[5] R. S. Palais, "The principle of symmetric criticality, Communications in Mathematical Physics, vol. 69, no. 1, pp. 19-30, 1979.

[6] M. Struwe, Variational Methods, vol. 34 of Results in Mathematics and Related Areas, Springer, Berlin, Germany, 3rd edition, 2000.

[7] I. Gelfand and S. Formin, Calculus of Variations, Nauka, Moscow, Russia; Prentice-Hall, Englewood Cliffs, NJ, USA, English edition, 1965. 


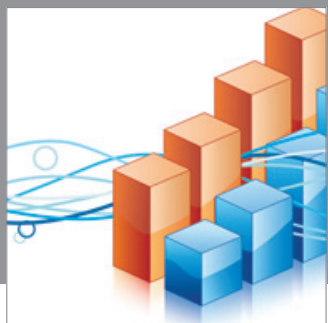

Advances in

Operations Research

mansans

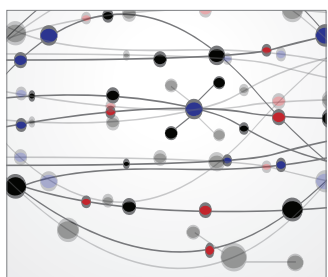

The Scientific World Journal
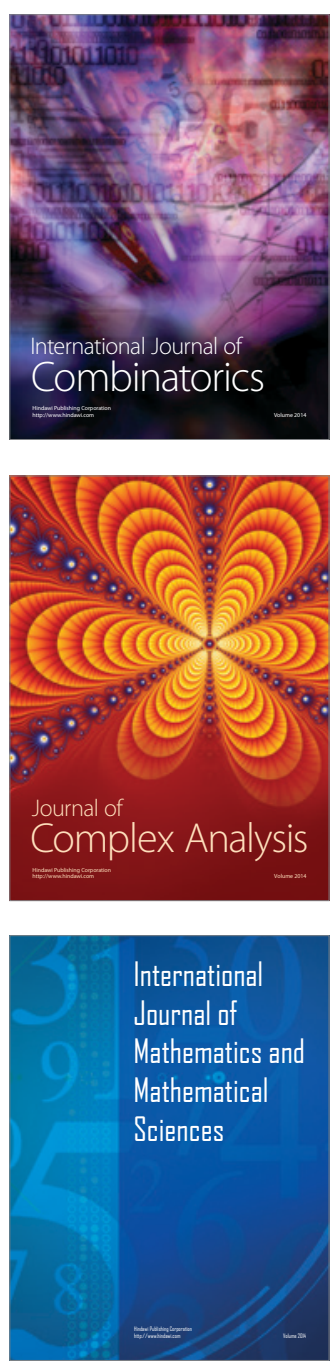
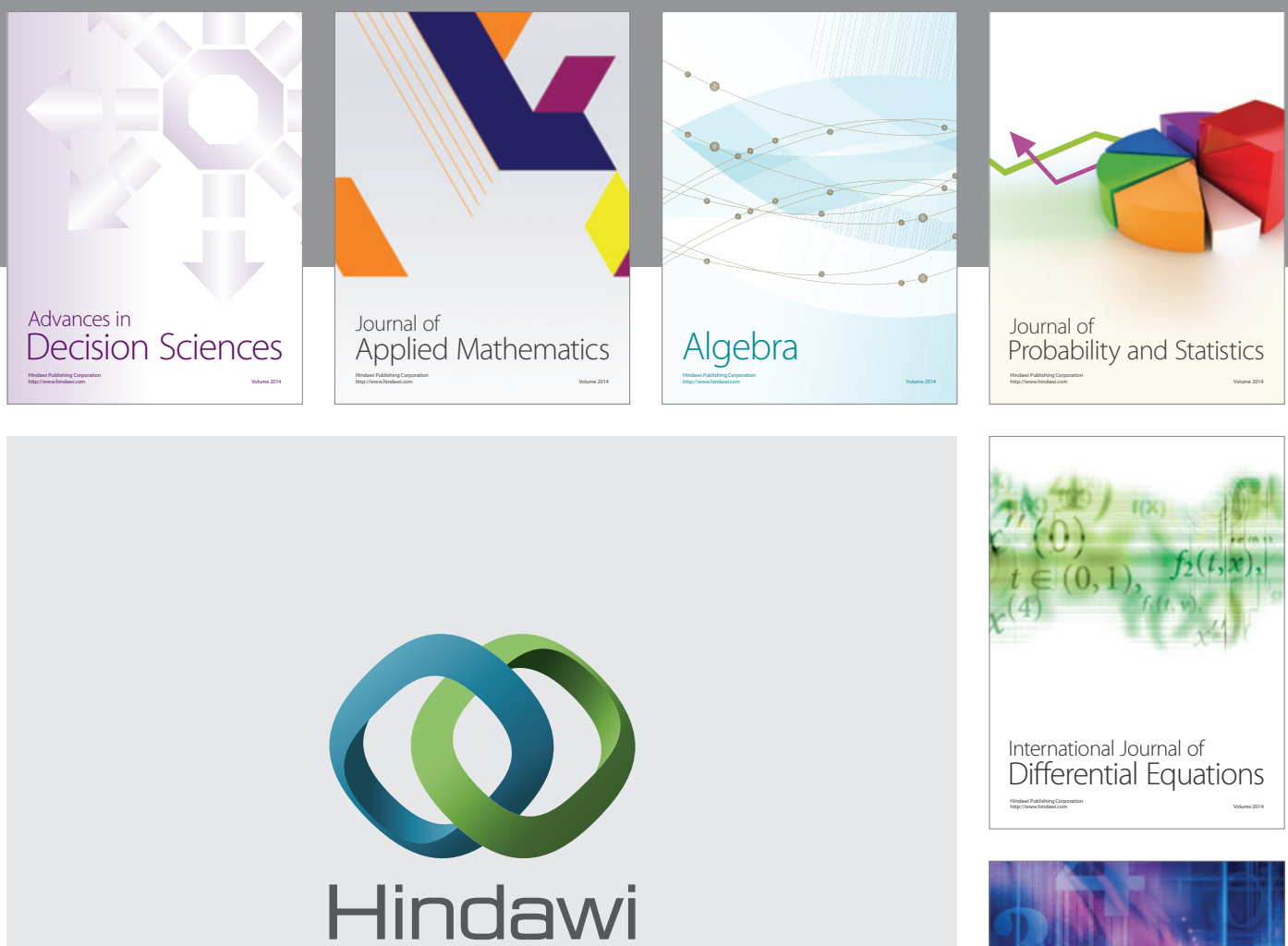

Submit your manuscripts at http://www.hindawi.com
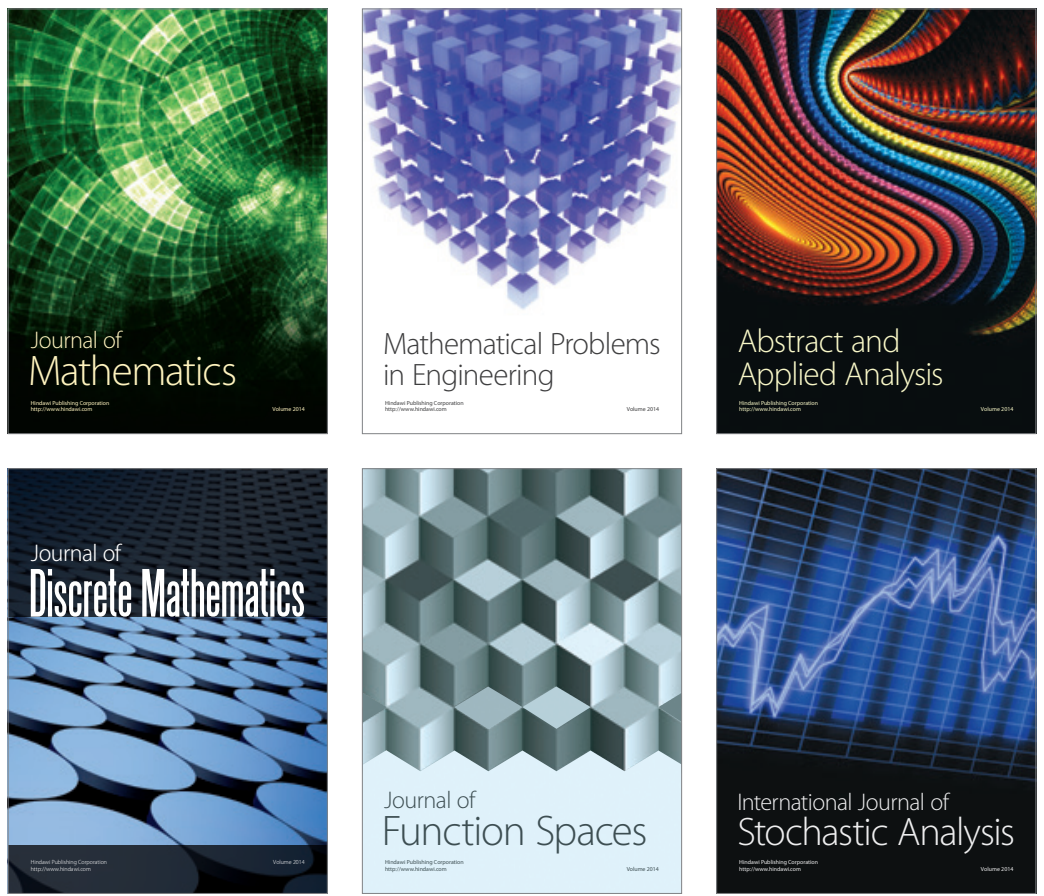

Journal of

Function Spaces

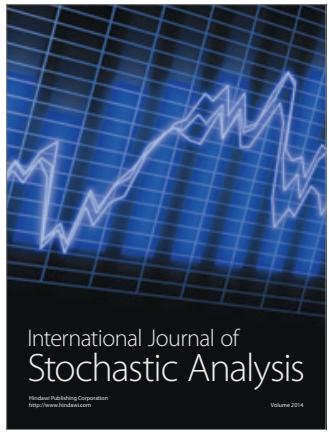

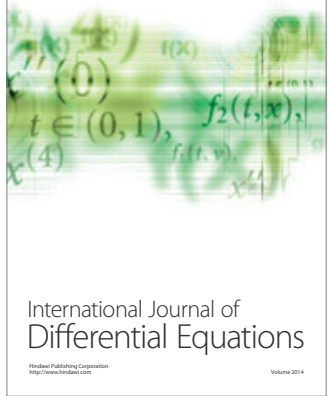
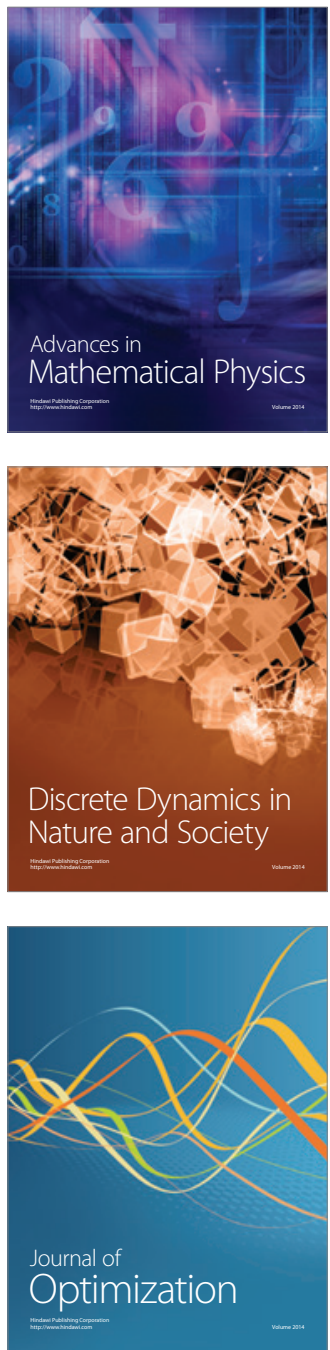